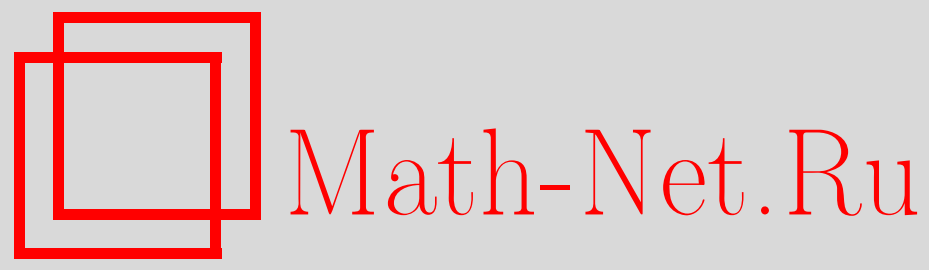

В. Л. Попов, О замкнутости некоторых орбит алгебраических групп, Функи. анализ и его прил., 1997, том 31, выпуск 4, 76-79

DOI: https://doi.org/10.4213/faa496

Использование Общероссийского математического портала MathNet.Ru подразумевает, что вы прочитали и согласны с пользовательским соглашением

http://www . mathnet.ru/rus/agreement

Параметры загрузки:

IP : 3.91 .87 .62

26 апреля 2023 г., 18:32:52

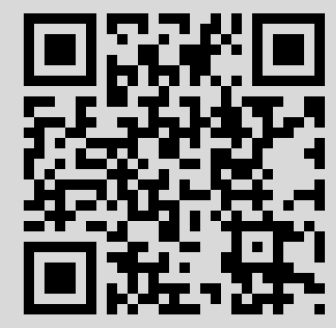




\title{
О замкнутости некоторых орбит алгебраических групп ${ }^{\star}$
}

\author{
(C) 1997. В. Л. Попов
}

В. И. Арнольду к его шестидесятилетию

В заметке доказывается критерий замкнутости некоторых орбит алгебраических групп.

Отметим два обстоятельства. Во-первых, большинство известных результатов о замкнутости орбит относится к случаю нулевой характеристики основного алгебраически замкнутого поля $k$, см. [6]. Мы не налагаем на характеристику поля $k$ никаких ограничений. Вопрос о характеристике важен для приложений. Так, пользуясь настоящим критерием, автор нашел естественное задание образующими и соотношениями аффинных координатных алгебр связных редуктивных алгебраических групп, годное в любой характеристике ([5]; в 1995 г. это было предметом докладов автора на сессии AMS в Чикаго и конференциях в Реймсе и Кортоне). Существование такого задания дает надежду найти квантование указанных алгебр в положительной характеристике (в нулевой это было сделано в [3]). Во-вторых, большинство известных результатов о замкнутости орбит относится к редуктивным группам, см. [6]. Настоящий критерий применим к более широкому классу групп.

1. Далее рассматриваются только линейные алгебраические группы. Помимо стандартных, см. $[2,6]$, используются следующие обозначения:

$\mathfrak{X}(H)$ - группа характеров алгебраической группы $H$ в аддитивной записи;

$h^{\lambda}$ - значение характера $\lambda \in \mathfrak{X}(H)$ на $h \in H$;

$\mathbb{Q} \geqslant 0:=\{r \in \mathbb{Q} \mid r \geqslant 0\}$.

2. Основным результатом заметки является следующая

Теорема 1. Пусть $R$ - алгебраическал группа, $S$ - ее борелевскал подгруппа и $T$ - максимальный тор в $S$. Предположим, что существуют характеры $\alpha_{1}, \ldots, \alpha_{d} \in \mathfrak{X}(T)$ (не обязательно все различные) и гомоморфизмьи $\varphi_{i}: \mathbb{G}_{a} \rightarrow S, i=1, \ldots, d$, обладаюшие следуюшими свойствами:

(a) $s \varphi_{i}(t) s^{-1}=\varphi_{i}\left(s^{\alpha_{i}} t\right)$ для всех $s \in T, t \in \mathbb{G}_{a}$;

(b) $S$ порождена $T$ и всеми $\varphi_{i}\left(\mathbb{G}_{a}\right)$;

(c) выпуклая оболочка множества $\Delta:=\left\{\alpha_{1}, \ldots, \alpha_{d}\right\}$ в $\mathfrak{X}(T) \otimes_{\mathbb{Z}} \mathbb{Q}$ не содержит нуля.

Пусть $V$ - прямая сумма конечномерных алгебраических $R$-модулей $V_{1}, \ldots$, $V_{m}$ u $v_{i} \in V_{i}$ - весовой вектор тора $T$ с весом $\lambda_{i} \in \mathfrak{X}(T)$. Тогда следуюшие свойства вектора $v=v_{1}+\cdots+v_{m} \in V$ эквивалентны:

(i) орбита $R \cdot v$ замкнута в $V$;

(ii) орбита $S \cdot v$ замкнута в $V$;

* Автор благодарит за поддержку Международный институт математической физики им. Э. Шрёдингера (Вена) и CRDF (грант No. RM1-206). 
(iii) орбита $T \cdot v$ замкнута в $V$;

(iv) нуль - внутренняя точка выпуклой оболочки множества $\left\{\lambda_{1}, \ldots, \lambda_{m}\right\}$ в $\mathfrak{X}(T) \otimes_{\mathbb{Z}} \mathbb{Q}$.

ЗАмЕчАниЕ 1. Если char $k=0$, то система характеров $\left\{\alpha_{i}\right\}$ и гомоморфизмов $\left\{\varphi_{i}\right\}$, обладающих свойствами (a) и (b), всегда существует, см. [4].

СлЕдСТВИЕ 1. Пусть $G$ - редуктивная алгебраическая группа, $T-e е$ максимальный тор и $R$ - замкнутая подәруппа в $G$, содержашая $T$. Пусть $V$ - прямая сумма конечномерных алгебраических $R$-модулей $V_{1}, \ldots, V_{s} u$ $v_{i} \in V_{i}$ - весовой вектор тора $T$ с весом $\lambda_{i} \in \mathfrak{X}(T)$. Тогда следуюшие свойства вектора $v=v_{1}+\cdots+v_{s} \in V$ эквивалентны:

(i) орбита $R \cdot v$ замкнута в $V$;

(ii) орбита $T \cdot v$ замкнута в $V$;

(iii) нуль - внутренняя точка вылуклой оболочки множества $\left\{\lambda_{1}, \ldots, \lambda_{s}\right\}$ в $\mathfrak{X}(T) \otimes_{\mathbb{Z}} \mathbb{Q}$.

ДокАЗАТЕЛЬСТво СЛЕДСТВИЯ 1 . Как известно [2], в этом случае $S$ порождена тором $T$ и содержащимися в $S$ одномерными унипотентными корневыми подгруппами, причем при соответствующем выборе системы простых корней в $\Delta$ входят только положительные корни. Поэтому условия теоремы 1 выполнены.

СлЕДСТВИЕ 2. Если в условиях следствия 1 каждый $R$-модуль $V_{i}$ является G-модулем, а $P$ и $B$ - соответственно параболическая и борелевская подгруппьл в $G$, содержащие $T$, то свойства (i), (ii), (iii) эквивалентнь следуюиим:

(iv) орбита $B \cdot v$ замкнута в $V$;

(v) орбита $P \cdot v$ замкнута в $V$;

(vi) орбита $G \cdot v$ замкнута в $V$.

3. Доказательство теоремы 1 опирается на следующий общий критерий замкнутости орбиты связной разрешимой алгебраической группы преобразований $H$ аффинного алгебраического многообразия $X$, доказанный в [4].

Пусть $x$ - точка многообразия $X$. Рассмотрим орбитный морфизм $\nu: H \rightarrow$ $X, h \mapsto h \cdot x$, и соответствующий коморфизм $\nu^{*}: k[X] \rightarrow k[H]$. Множество

$\mathfrak{X}(H)_{X, x}:=\left\{\lambda \in \mathfrak{X}(H) \mid\right.$ функция $H \rightarrow k, h \mapsto h^{\lambda}$, содержится в $\left.\nu^{*}(k[X])\right\}$

является подмоноидом в $\mathfrak{X}(H)$.

ТеОРема 2. Следуюиие свойства эквивалентны:

(i) орбита $H \cdot x$ замкнута в $X$;

(ii) моноид (1) является группой.

ЗАмечАниЕ 2. Рассмотрим непустое подмножество $\mathfrak{S}$ конечномерного векторного пространства $L$ над $\mathbb{Q}$. Пусть $\mathbb{Q} \geqslant 0 \mathfrak{S}$, conv $\mathfrak{S}$ и $\mathbb{Q} \mathfrak{S}$ - соответственно выпуклый конус, порожденный $\mathfrak{S}$, выпуклая оболочка и линейная оболочка подмножества $\mathfrak{S}$ в $L$. Тогда следующие свойства эквивалентны:

(i) нуль - внутренняя точка conv $\mathfrak{S}$;

(ii) $\mathbb{Q} \geqslant 0 \mathfrak{S}=\mathbb{Q} \mathfrak{S}$.

Если $\mathfrak{S}$ является подполугруппой векторной группы $L$, то (i) и (ii) эквивалентны свойству

(iii) $\mathfrak{S}$ является группой. 
Это утверждение известно специалистам, но мне не удалось найти ссылку. Доказательство несложно.

4. ДоКАЗАТЕЛЬСТВО ТЕОРЕмы 1 . ОТождествим $\mathfrak{X}(S)$ с $\mathfrak{X}(T)$ с помощью изоморфизма ограничения функций и обозначим эту группу через $\mathfrak{X}$.

(iii) $\Leftrightarrow($ iv). Это частный случай критерия Гильберта-Мамфорда, см. [6, п. 5.3] (впрочем, поскольку $\left\{\lambda_{1}, \ldots, \lambda_{s}\right\}$ - система образующих моноида $\mathfrak{X}(T)_{V, v}$, утверждение следует из теоремы 2 и замечания 2).

(ii) $\Rightarrow$ (i). Это вытекает из того, что $R / S$ полно и $R \cdot v=R \cdot(S \cdot v)$.

(i) $\Rightarrow$ (iv). Предположим, что $R \cdot v$ замкнута в $V$. Если (iv) не выполнено, то найдется такая линейная функция $l \in\left(\mathfrak{X} \otimes_{\mathbb{Z}} \mathbb{Q}\right)^{*}$, что $n_{i}:=l\left(\lambda_{i}\right) \in \mathbb{Q} \geqslant 0$ для всех $i$ и по крайней мере одно из этих чисел, скажем, $n_{1}$, положительно. Умножая $l$ на подходящее положительное целое число, можно считать, что $l(\mathfrak{X}) \subseteq \mathbb{Z}$. Следовательно, существует такой гомоморфизм $\gamma: \mathbb{G}_{m} \rightarrow T$, что $\gamma(t) \cdot v=$ $\sum_{i=1}^{s} t^{n_{i}} v_{i}$. Поэтому замыкание $T \cdot v$ в $V$ содержит $u:=\sum_{i=1}^{s} u_{i}$, где $u_{i}=v_{i}$, если $n_{i}=0$, и $u_{i}=0$, если $n_{i}>0$. Поскольку орбита $R \cdot v$ замкнута в $V$, это замыкание содержится в $R \cdot v$. С другой стороны, $u \notin R \cdot v$, так как $u_{1}=0$, а $v_{1} \neq 0$, - противоречие.

(iv) $\Rightarrow$ (ii). Предположим, что выполнено (iv). Пусть $S^{u}$ - унипотентный радикал $S$.

Если $L$ - конечномерный алгебраический $R$-модуль и $x \in L_{\lambda}, x \neq 0$, то из (a) следует, что $\varphi_{i}(t) \cdot x=x+t x_{1}+t^{2} x_{2}+\ldots$, где $x_{m} \in L_{\lambda+m \alpha_{i}}$ (только конечное число $x_{i}$ отлично от нуля). Ввиду (b) группа $S^{u}$ порождена всеми $\varphi_{i}\left(\mathbb{G}_{a}\right), i=$ $1, \ldots, d$. Из (c) вытекает, что любая сумма элементов из $\Delta$ является ненулевым элементом из $\mathfrak{X}$. Поскольку $S=T S^{u}$, из этих фактов следует, что если $s \in S$, то $s \cdot x=s^{\lambda} x+x^{\prime}$, где $x^{\prime}$ — вектор, в разложении которого в сумму весовых векторов тора $T$ не содержится слагаемого веса $\lambda$.

Применяя это к $L=V_{i}, x=v_{i}$, получаем для любого $s \in S$

$$
s \cdot v_{i}=s^{\lambda_{i}} v_{i}+v_{i}^{\prime}, \quad v_{i}^{\prime} \in V_{i}
$$

где $v_{i}^{\prime}$ обладает тем свойством, что его разложение в сумму весовых векторов тора $T$ не содержит слагаемых веса $\lambda_{i}$.

Зафиксируем в $V_{i}$ такой базис из весовых векторов тора $T$, чтобы его первым вектором был $v_{i}$, и пусть $f_{i}$ - первый вектор дуального базиса в $V_{i}^{*}$. Отождествим естественным образом $V_{i}^{*}$ с линейным подпространством в $V^{*}$. Тогда $f_{i}\left(v_{i}\right)=1, f_{i}\left(v_{j}\right)=0$ при $i \neq j$ и $f_{i}\left(v_{j}^{\prime}\right)=0$ для всех $i$ и $j$.

Отсюда и из (2) вытекает, что для любого $s \in S$

$$
f_{i}(s \cdot v)=f_{i}\left(\left(s^{\lambda_{1}} \cdot v_{1}+v_{1}^{\prime}\right)+\cdots+\left(s^{\lambda_{s}} \cdot v_{s}+v_{s}^{\prime}\right)\right)=s^{\lambda_{i}}, \quad s \in S .
$$

Рассмотрим полугруппу (1) для $H=S, X=V, x=v$. Ввиду (3)

$$
\lambda_{i} \in \mathfrak{X}_{V, v}:=\mathfrak{X}(S)_{V, v} .
$$

Пусть $C$ - выпуклый конус, порожденный $\left\{\lambda_{1}, \ldots, \lambda_{s}\right\}$, и $L-$ его линейная оболочка в $\mathfrak{X} \otimes_{\mathbb{Z}} \mathbb{Q}$. Ввиду (iv) и замечания 2

$$
C=L .
$$


Из (4) и (5) получаем

$$
L \subseteq \mathbb{Q} \geqslant 0 \mathfrak{X}_{V, v} .
$$

Пусть, как обычно, $S_{v}$ обозначает $S$-стабилизатор точки $v$ и $T_{v}=T \cap S_{v}$. Из определения $\mathfrak{X}_{V, v}$ следует, что

$$
s^{\lambda}=1 \text { для любого } s \in S_{v} \text { и } \lambda \in \mathfrak{X}_{V, v} .
$$

В частности, это верно для $s \in T_{v}$.

С другой стороны, из определения $v$ вытекает, что

$$
T_{v}=\left\{t \in T \mid t^{\lambda_{i}}=1 \text { для всех } i\right\} .
$$

Поскольку $\operatorname{dim} \mathbb{Q} \mathfrak{S}=\operatorname{codim}_{T}\left\{t \in T \mid t^{\lambda}=1, \lambda \in \mathfrak{S}\right\}$ для любого непустого подмножества $\mathfrak{S}$ в $\mathfrak{X}$, из $(7)$ и (8) следует, что

$$
\operatorname{dim} \mathbb{Q} \mathfrak{X}_{V, v} \leqslant \operatorname{dim} L .
$$

Теперь из (6) и (9) вытекает, что $\mathbb{Q} \geqslant 0 \mathfrak{X}_{V, v}=\mathbb{Q X}_{V, v}$, и поэтому $\mathfrak{X}_{V, v}$ является группой (см. замечание 2). Следовательно, орбита $S \cdot v$ замкнута в $V$ по теореме 2.

ЗАмеЧАнИЕ. При $k=\mathbb{C}$ импликацию (ii) $\Rightarrow$ (vi) из следствия 2 теоремы 1 можно доказать также и трансцендентными методами. Рассуждения, с точностью до небольшой модификации, те же, что и в доказательстве предложения 1.2 в [1] (где устанавливается замкнутость орбит иного вида).

А именно, пусть $K$ - максимальная связная компактная подгруппа в $G$ и $(, \quad)-K$-инвариантное эрмитово скалярное произведение на $V$, относительно которого $V_{i}$ и $V_{j}, i \neq j$, ортогональны. Поскольку $T \cdot v$ замкнута в $V$, функщия $v \rightarrow(v, v)$ достигает на $T \cdot v$ минимума. Так как $t \cdot v_{i} \in V_{\lambda_{i}}$ при $t \in T$, можно, изменив обозначения, считать, что этот минимум достигается на $v$. Значит, $((\operatorname{Lie} T) \cdot v, v)=0$. Так как $(\operatorname{Lie} G)_{\alpha} \cdot\left(V_{i}\right)_{\lambda_{i}} \subseteq\left(V_{i}\right)_{\alpha+\lambda_{i}}$ для любого корня $\alpha$, то из ортогональности весовых подпространств разных весов и ортогональности различных подпространств $V_{i}$ следует, что $\left((\operatorname{Lie} G)_{\alpha} \cdot v, v\right)=0$. Это показывает, что $((\operatorname{Lie} G) \cdot v, v)=0$. Замкнутость орбиты $G \cdot v$ в $V$ вытекает теперь из критерия Кемпфа-Несс (см. [6, п. 6.12]).

\section{ЛитератУРА}

1. Dadok J., Kac V. J. Algebra, 92, 504-524 (1985). 2. Хамфри Д. Линейные алгебраические группы. Наука, M., 1980. 3. Joseph A. Quantum groups and their primitive ideals, Springer-Verlag, 1995. 4. Попов В. Л. Матем. сб., 135, o. 3, 385402 (1988). 5. Popov V. L. Generators and relations of the affine coordinate rings of the connected semisimple algebraic groups. Preprint (1995). 6. Винберг Э. Б. и Попов В. Л. Теория инвариантов. Итоги науки и техники. Совр. пробл. матем. Фунд. направл. Алгебра-4, ВИНИТИ, Т. 55, 1989, с. 137-309.

Московский государственный институт

Поступило в редакцию электроники и математики 6 февраля 1997 г. e-mail: popov@ppc.msk.ru 